\section{"A medida da fome": as escalas psicométricas de insegurança alimentar e os povos indígenas no Brasil}

\author{
"Measuring hunger": psychometric scales of food \\ insecurity and indigenous peoples in Brazil
}
"La medida del hambre": escalas psicométricas de inseguridad alimentaria y pueblos indígenas
en Brasil

Adriana Romano Athila 1

Maurício Soares Leite 2

doi: 10.1590/0102-311X00208019

\section{Resumo}

Validada para o contexto nacional brasileiro em 2004, a Escala Brasileira de Insegurança Alimentar (EBIA) tem desde então avaliado e mensurado a experiência de insegurança alimentar de domicílios rurais e urbanos, inicialmente na Pesquisa Nacional por Amostra de Domicílios e posteriormente na Pesquisa Nacional de Demografia e Saúde. No entanto, os povos indigenas não foram especificamente examinados nesses levantamentos, a despeito do reconhecimento das suas vulnerabilidades alimentar e nutricional. Nesse cenário, analisamos e discutimos a aplicação da EBIA entre povos indígenas do país, com base em um conjunto de questões aqui consideradas fundamentais para a compreensão e a mensuração de sua experiência de insegurança alimentar e "fome". É realizada uma análise sociopolítica e etnográfica de um conjunto de documentos oficiais e artigos significativos sobre o uso de escalas psicométricas de insegurança alimentar entre povos indígenas brasileiros, em contraste com artigos internacionais sobre a validação e aplicação das escalas em outros contextos socioculturais. As iniciativas de adaptação e aplicação da EBIA aos contextos indígenas brasileiros indicam que compreender e mensurar a insegurança alimentar entre esses povos é um desafio de magnitude considerável. Particularmente complexa é a proposta de garantir a comparabilidade entre contextos distintos sem deixar de contemplar as plurais singularidades locais. Propomos que estudos etnográficos constituam componentes específicos de futuras iniciativas dedicadas ao tema, e que contemplem aspectos como a sazonalidade da produção de alimentos, seus processos diferenciais de monetarização e o dinamismo de seus sistemas alimentares.

\author{
Correspondência \\ A. R. Athila \\ Av. Ayrton Senna 270, bloco 2, apto. 2007, Rio de Janeiro, RJ \\ 22793-000, Brasil. \\ adrianarathila@gmail.com \\ 1 Escola Nacional de Saúde Pública Sergio Arouca, Fundação \\ Oswaldo Cruz, Rio de Janeiro, Brasil. \\ 2 Programa de Pós-graduação em Nutrição, Universidade \\ Federal de Santa Catarina, Florianópolis, Brasil.
}




\section{Introdução}

A vulnerabilidade da situação de saúde de povos indígenas vem sendo sistematicamente reconhecida em todo o mundo, em um quadro em que são evidentes os indicadores de saúde, alimentação e nutrição amplamente desfavoráveis, e sistematicamente piores que aqueles registrados nos segmentos não indígenas da população 1,2. No Brasil o quadro não é distinto. Em termos gerais, o perfil epidemiológico indígena é também caracterizado pela desigualdade frente ao segmento não indígena, e pelo predomínio de doenças infecciosas e parasitárias como causas de morbidade e mortalidade 3 . Contemporaneamente, novos agravos, como doenças crônicas não transmissíveis e causas externas, são responsáveis por uma proporção crescente da morbimortalidade 3,4.

No que se refere especificamente aos indicadores nutricionais, os registros disponíveis apontam para um quadro igualmente complexo, em que prevalências de moderadas a elevadas de déficits estaturais e ponderais entre menores de cinco anos coexistem com registros cada vez mais frequentes de sobrepeso e obesidade entre adultos e adolescentes, e por vezes também entre crianças 5,6,7. A anemia é também um problema de saúde pública entre crianças e mulheres em idade reprodutiva 8,9. Para além dos tradicionais indicadores de alimentação e nutrição, diversos autores defendem a necessidade de identificar, compreender e, ao mesmo tempo, quantificar a experiência de insegurança alimentar dessas populações, como forma de informar e instrumentalizar gestores e políticas de diversos setores 10,11.

Diante da reconhecida dificuldade em definir e mensurar a fome 12,13, a proposta de elaboração de uma escala psicométrica de insegurança alimentar aconteceu originalmente nos anos 1990, no contexto estadunidense. Seu objetivo primordial era quantificar como especificamente grupos populacionais considerados "mais vulneráveis" compreendiam e experienciavam o fenômeno da "insegurança alimentar" e da "fome" 13,14. Ela surgiu na forma de questionários com o objetivo de avaliar impactos de políticas públicas de alimentação e nutrição sobre grupos específicos da população. Ali, o instrumento atualmente utilizado pelo Departamento da Agricultura foi desenvolvido por meio de pesquisas transversais de validação, com duração de aproximadamente uma década. A construção de sua validade envolveu o contato direto dos pesquisadores com os diferentes segmentos da população nacional. Rigorosas pesquisas em campo, na profundidade temporal, buscavam contornar a potencial transitoriedade de eventos capazes de comprometer o acesso ou incertezas com relação à sua comida 14 .

No Brasil, onde a segurança alimentar e nutricional é atualmente definida como a "realização do direito de todos ao acesso regular e permanente a alimentos de qualidade, em quantidade suficiente, sem comprometer o acesso a outras necessidades essenciais, tendo como base práticas alimentares promotoras de saúde que respeitem a diversidade cultural e que sejam ambiental, cultural, econômica e socialmente sustentáveis" 15 , entre 2003 e 2004 uma versão do instrumento, a Escala Brasileira de Insegurança Alimentar (EBIA), foi adaptada e validada para uso no país 16.

A EBIA é atualmente composta por 14 perguntas 17. Essas perguntas buscam entender o fenômeno, visto como progressivamente mais grave, com base em aspectos da experiência da insegurança alimentar que seriam comuns a outros contextos socioculturais: a ansiedade ou dúvida sobre a disponibilidade futura de alimentos; o comprometimento da qualidade dos alimentos; o comprometimento da quantidade de alimentos, inicialmente entre adultos, e progressivamente atingindo as crianças; e a fome.

Já em 2004, a EBIA foi aplicada na Pesquisa Suplementar de Segurança Alimentar, por meio da Pesquisa Nacional por Amostra de Domicílios (PNAD) 18. Em 2006, a EBIA passou a integrar a Pesquisa Nacional de Demografia em Saúde (PNDS) 19. Desde então, a escala, definida como um "instrumento robusto de avaliação", vem servindo para avaliar a insegurança alimentar de domicílios rurais e urbanos, subsidiando gestores na identificação de segmentos vulneráveis da população e também na avaliação e ajuste de políticas públicas diversas 16 . No entanto, indígenas e outras minorias étnicas e populacionais, a despeito de sua vulnerabilidade alimentar e nutricional, não foram especificamente examinados nesses levantamentos.

Debates sobre rendimento e uso das escalas em contextos diversos daquele original e os procedimentos metodológicos necessários à sua adequação às especificidades locais têm se multiplicado 14,20,21,22. Assume-se que a validação não pode ser uma "simples tradução", ainda que "qualificada" 20 , e que o instrumento deve demonstrar um balanço entre a singularidade da experiência da insegurança alimentar e da fome e as evidências capazes de apontar as dimensões comuns desta experiência. 
Descrito como um desafio que precisa ser enfrentado 10 , o uso da EBIA entre povos indígenas é objeto de um número ainda restrito de publicações 23,24,25,26. Tendo como pano de fundo o diálogo e a aproximação constitucionalmente prescritos entre os modos de vida de povos indígenas e a formulação, a execução e sua participação na governança de políticas de nutrição e alimentação, o presente estudo se propõe a examinar, por meio de uma abordagem analítica de documentos e outras modalidades de produção bibliográfica, alguns conceitos, pressupostos, procedimentos metodológicos e de participação indígena envolvidos na recente trajetória do desenvolvimento, validação e de algumas experiências de aplicação da EBIA entre povos indígenas brasileiros específicos.

\section{Metodologia}

Foi realizada uma análise sociopolítica e etnográfica de um conjunto de documentos oficiais e artigos selecionados sobre a validação de escalas psicométricas de insegurança alimentar em contextos socioculturais diversos e entre povos indígenas brasileiros, de acordo com a sugestão de Merry 27. A autora investiga etnograficamente como indicadores destinados a quantificar fenômenos fundamentalmente sociais são construídos, os efeitos deste modo de construção sobre políticas públicas e outras esferas mais ou menos formais de governança e decisões, e seu comprometimento com os "mundos sociais e culturais" de atores, organizações, campos disciplinares e de poder dos quais são derivados ou com base nos quais são geridos 27 . A análise aborda como indígenas e suas especificidades têm sido representados em procedimentos que envolvem quantificação, conforme análises recentes do campo da sociodemografia vêm adotando no Brasil 28,29. Com ênfase em algumas experiências de aplicação e validação da EBIA entre povos indígenas brasileiros, são abordadas e discutidas as ideias fundantes da genealogia do instrumento, com referência a aspectos sociopolíticos de seu contexto original de aplicação e recomendações para o uso em universos socioculturalmente diversos.

\section{O desenvolvimento da EBIA entre povos indígenas no Brasil}

Com a constituição do Sistema Nacional de Segurança Alimentar e Nutricional (SISAN), a segurança alimentar e nutricional passou a ser um direito reconhecido como Direito Humano à Alimentação Adequada (DHAA) 15. Posteriormente, esses conceitos foram expressos na Política Nacional de Segurança Alimentar e Nutricional 30. No tocante a povos indígenas e às determinações do SISAN, a produção específica de informações, como quaisquer políticas, programas e ações intersetoriais relacionadas à segurança alimentar e nutricional e ao DHAA, devem observar sua diversidade sociocultural e ambiental e particularidades regionais 15,31 . Como acontece com outros indicadores sociodemográficos e de saúde, dados disponíveis sobre a segurança alimentar e nutricional de povos indígenas brasileiros contrastam significativamente com o corpus produzido sobre o restante da população, regularmente avaliada desde a validação da EBIA, ainda na década de 2000.

Poucos trabalhos dialogam com a adequação do instrumento, quando deslocado para um contexto de singular diversidade. Eles têm caraterísticas particulares, dos critérios de seleção e variabilidade dos grupos estudados ao modo de construção metodológico-conceitual e procedimentos de aplicação do instrumento.

De um conjunto de 305 etnias indígenas oficialmente reconhecidas pelo Estado 32, a aplicação propriamente dita de alguma versão da EBIA foi feita entre os Terena 23 e, sobretudo, entre os subgrupos ou parcialidades Guarani 24,25,33,34, povos sobre os quais há uma extensa literatura clássica e contemporânea 35. Estudos prévios ou complementares foram realizados também junto aos Guarani 10,26 e, ainda, entre os Kanamari e Kulina, do Amazonas 31.

Antes que uma revisão bibliográfica exaustiva, selecionamos alguns trabalhos que permitem refletir tanto sobre dimensões conceituais e metodológicas consideradas cruciais, à luz daqueles marcos regulatórios, quanto das considerações pertinentes ao desenvolvimento e aplicação de escalas de insegurança alimentar em contextos socioculturalmente diversos - algo especialmente heterogêneo no caso de povos indígenas brasileiros. São diversas situações de terras, ecossistemas e graus de degradação, históricos de contato, modos de interação com não indígenas, com o mercado e a economia 
regionais. Essas variáveis, por sua vez, articulam-se de formas potencialmente diferentes à pluralidade linguístico-cultural e das modalidades de parentesco e organização social desses povos.

O Quadro 1 permite uma visualização sistemática de aspectos-chave dos estudos selecionados, como etnias envolvidas e modalidades da participação de indígenas e comunidades nas fases das pesquisas. Especialmente relevantes à análise são as informações sobre etapas e procedimentos metodológico-conceituais adotados, sobretudo sob a perspectiva qualitativa. Por essa razão, o Quadro 1 não contempla diagnósticos da situação de insegurança alimentar decorrentes da aplicação das escalas.

Na Região Centro-oeste, Estado de Mato Grosso do Sul, participam dos estudos três comunidades Terena 23 e os Guarani de três comunidades Guarani e Kaiowá 24. No Sudeste, Estado de São Paulo, participam os Guarani de quatro comunidades Nandeva, Tupi-Guarani e Mbya 10,25,26,33 e, no Espírito Santo, uma comunidade Mbya 34. Na Região Norte, três comunidades Kulina e Kanamari contam com estudos fundamentalmente conceituais 31 .

Quanto às características fundiárias das comunidades-alvo, as Guarani têm status peculiar. Das oito aldeias participantes dos diferentes estudos, três delas - as Guarani e Kaiowá de Mato Grosso do Sul - estão em áreas de retomada 24. Das cinco comunidades restantes, quatro estão em Terras Indígenas (TI) regularizadas, próximas 34 ou relativamente próximas 26,33 a centros urbanos. Uma delas estava em TI não regularizada e em zona periurbana 25,33. Os Terena, de Mato Grosso do Sul 23, e os Kulina e os Kanamari, do Estado do Amazonas, estão em TIs regularizadas, estes últimos em TIs distantes cerca de $1.200 \mathrm{~km}$ em linha reta da cidade de Manaus 31 .

No ano de 2005, imediatamente após a validação da EBIA, já havia pesquisas que estendiam seu uso a povos indígenas ${ }^{3}$. O estudo que envolveu a aplicação de um instrumento derivado da EBIA, entre os Terena 23 , inaugurou as publicações específicas sobre o tema no Brasil. A pesquisa envolveu visitas ao longo de nove meses, compreendendo 49 famílias com crianças menores de cinco anos, de três das oito aldeias da TI. A aplicação da EBIA foi precedida por etapas metodológicas de adaptação bastante marcadas e que se replicam nos demais estudos selecionados, obedecendo a ordenamentos, modos de organização e denominação muito semelhantes. Sobre o estudo de Franceschini 24 não há informações acerca de procedimentos e adaptações do instrumento, referido ora por EBIA, ora por "EBIA indígena".

Um primeiro passo comum é a realização do painel de especialistas, com pesquisadores não indígenas que predominantemente trabalham com o tema da segurança alimentar e das condições de saúde e nutrição de povos indígenas. Posteriormente ao painel, do qual resulta outra versão prévia da EBIA, à exceção de Vargas et al. 34, os estudos invariavelmente realizam grupos focais com características e número variáveis. Nos painéis, os especialistas colocam diferentes tipos de desafios à aplicação da EBIA em contextos indígenas brasileiros. A constatação de sua "sociodiversidade" é uma objeção constante, ao lado de variáveis regionais, como a sazonalidade 31.

O estudo de Fávaro et al. 23 realiza um grupo focal sediado em uma das aldeias, com oito participantes indígenas selecionados com o auxílio do agente de saúde indígena (AIS). A EBIA sofre pequenas adaptações e é reapresentada ao grupo, mantendo seu número original de perguntas, em português. Houve mudança na linguagem, para uma compreensão facilitada, reduzindo o período de referência das perguntas de segurança alimentar e nutricional de três para um mês. Uma particularidade dos resultados é que mesmo domicílios considerados seguros apresentavam uma dieta nutricionalmente deficiente. Os autores consideram que escalas devem ser desenvolvidas e adaptadas aos diferentes povos indígenas do país, advertindo que o instrumento fora adaptado especificamente para os Terena.

Yuyama et al. 31 submetem as questões aplicadas à população rural brasileira ao painel de especialistas. Das discussões deriva uma versão da EBIA em português, mas com "pequenas adaptações de linguagem”, posteriormente apresentada a três grupos focais realizados nas três comunidades, com 18 participantes indígenas. A escala é discutida em português, provocando incompreensões aos indígenas - como por exemplo, o conceito de "estratégia" -, atribuídas aos altos índices de analfabetismo, mas não a incompatibilidades linguísticas ou conceituais. Em contrapartida, o estudo demonstra uma singular abertura à epistemologia dos conceitos nativos relativos às diversas dimensões da insegurança alimentar, esboçando a produtividade de seu diálogo com a vida e as compreensões Kulina e Kanamari sobre sua comida. Recomendando evitar a adoção de conceitos de segurança alimentar e nutricional previamente às discussões com os indígenas, o estudo reafirma a importância da continui- 


\section{Quadro 1}

Uso da Escala Brasileira de Insegurança Alimentar (EBIA) ou suas versões entre povos indígenas brasileiros, em publicações selecionadas.

\begin{tabular}{|c|c|c|c|c|c|}
\hline Referência & Ano & Etnia (Estado) & Modalidade & $\begin{array}{l}\text { Comunidades envolvidas/ } \\
\text { famílias }\end{array}$ & Metodologia/validação/instrumento \\
\hline Fávaro et al. 23 & 2007 & $\begin{array}{l}\text { Terena (Mato } \\
\text { Grosso do Sul) }\end{array}$ & $\begin{array}{l}\text { Artigo } \\
\text { científico }\end{array}$ & $\begin{array}{l}49 \text { famílias de } 3 \\
\text { comunidades: Água Azul, } \\
\text { Olho d'Água e Oliveiras. }\end{array}$ & $\begin{array}{l}\text { Instrumento derivado/adaptação da EBIA; } \\
\text { painel de especialistas; um grupo focal, com } 8 \\
\text { participantes, em uma aldeia, selecionada com } \\
\text { a ajuda do agente indígena de saúde (AIS). }\end{array}$ \\
\hline Yuyama et al. 31 & 2008 & $\begin{array}{l}\text { Kulina e } \\
\text { Kanamari } \\
\text { (Amazonas) }\end{array}$ & $\begin{array}{l}\text { Artigo } \\
\text { científico }\end{array}$ & $\begin{array}{c}3 \text { comunidades: Cacau } \\
\text { (Kulina), Flexeira e Mamori } \\
\text { (Kanamari). }\end{array}$ & $\begin{array}{c}\text { Versão adaptada da EBIA; painel de } \\
\text { especialistas; um grupo focal por comunidade, } \\
\text { com } 18 \text { participantes indígenas ao todo. }\end{array}$ \\
\hline $\begin{array}{l}\text { Segall-Corrêa et } \\
\text { al. } 33\end{array}$ & 2009 & $\begin{array}{c}\text { Guarani } \\
\text { Ñandeva, } \\
\text { Tupi-Guarani e } \\
\text { Guarani Mbya } \\
\text { (São Paulo) }\end{array}$ & $\begin{array}{l}\text { Relatório final } \\
\text { de pesquisa }\end{array}$ & $\begin{array}{l}3 \text { comunidades de TI e } 1 \text { em } \\
\text { zona urbana: Rio Branco, } \\
\text { Rio Silveira, Piaçaguera e } \\
\text { Aldeinha. }\end{array}$ & $\begin{array}{l}\text { EBIA adaptada; painel de especialistas; três } \\
\text { grupos focais, com } 20 \text { pessoas de uma aldeia, } \\
\text { em uma pousada, visitas, entrevistas. }\end{array}$ \\
\hline Azevedo et al. 10 & 2009 & $\begin{array}{c}\text { Guarani } \\
\text { Ñandeva, } \\
\text { Tupi-Guarani, } \\
\text { Guarani Mbya } \\
\text { (São Paulo) }\end{array}$ & $\begin{array}{l}\text { Capítulo de } \\
\text { livro }\end{array}$ & $\begin{array}{l}3 \text { comunidades de três TI e } 1 \\
\text { em zona urbana: Rio Branco, } \\
\text { Rio Silveira, Piaçaguera, } \\
\text { Aldeinha. }\end{array}$ & $\begin{array}{c}\text { Descrições gerais sobre a pesquisa, seus } \\
\text { objetivos e sobre as aldeias envolvidas; não } \\
\text { aborda conceitos Guarani ou a metodologia } \\
\text { adotada. }\end{array}$ \\
\hline $\begin{array}{l}\text { Segall-Corrêa et } \\
\text { al. } 26\end{array}$ & 2010 & $\begin{array}{c}\text { Guarani } \\
\text { Ñandeva, } \\
\text { Tupi-Guarani, } \\
\text { Guarani Mbya } \\
\text { (São Paulo) }\end{array}$ & $\begin{array}{l}\text { Resumo de } \\
\text { congresso } \\
\text { em periódico } \\
\text { científico }\end{array}$ & $\begin{array}{l}4 \text { comunidades Guarani não } \\
\text { especificadas. }\end{array}$ & $\begin{array}{l}\text { EBIA adaptada; visitas às comunidades ao longo } \\
\text { de } 2 \text { anos; painel de especialistas; percepções } \\
\text { de insegurança alimentar exploradas em } \\
\text { entrevistas com membros de uma comunidade. }\end{array}$ \\
\hline Vargas et al. 34 & 2013 & $\begin{array}{l}\text { Guarani Mbya } \\
\text { (Espírito Santo) }\end{array}$ & $\begin{array}{l}\text { Artigo } \\
\text { científico }\end{array}$ & $\begin{array}{l}25 \text { domicílios de } 1 \text { aldeia: } \\
\text { Boa Esperança. }\end{array}$ & $\begin{array}{l}\text { "EBIA modificada"; painel de especialistas; } \\
\text { aplicada por entrevistadores treinados, } \\
\text { auxiliados por AIS. }\end{array}$ \\
\hline Franceschini 24 & 2016 & $\begin{array}{l}\text { Guarani Mbya } \\
\text { (Mato Grosso } \\
\text { do Sul) }\end{array}$ & $\begin{array}{l}\text { Resumo } \\
\text { executivo }\end{array}$ & $\begin{array}{l}75 \text { domicílios de } 3 \\
\text { comunidades em área de } \\
\text { retomada: Guaiviry, Ypo'i e } \\
\text { Kurusu Ambá. }\end{array}$ & $\begin{array}{c}\text { EBIA/EBIA Indígena. Não há } \\
\text { menção mais específica à metodologia. }\end{array}$ \\
\hline $\begin{array}{l}\text { Segall-Corrêa et } \\
\text { al. } 25\end{array}$ & 2018 & $\begin{array}{l}\text { Guarani Mbya } \\
\text { (São Paulo) }\end{array}$ & $\begin{array}{l}\text { Artigo } \\
\text { científico }\end{array}$ & $\begin{array}{l}3 \text { aldeias de Tl e } 1 \text { em zona } \\
\text { urbana: Rio Branco, Rio } \\
\text { Silveira, Piaçaguera. Testes } \\
\text { psicométricos aplicados em } \\
88 \text { domicílios em } 4 \text { aldeias: } \\
\text { Rio Silveira, Piaçaguera, Boa } \\
\text { Vista e Ubatuba *. }\end{array}$ & $\begin{array}{l}\text { EBIA-G validada após a testagem de duas } \\
\text { versões; painel com especialistas; } 3 \text { grupos } \\
\text { focais; não especifica aldeias participantes ou } \\
\text { local de realização; entrevistadores indígenas } \\
\text { aplicam a escala, colaboram em adaptações } \\
\text { junto ao grupo de pesquisa, discutindo os } \\
\text { resultados da pesquisa a eles apresentados. }\end{array}$ \\
\hline
\end{tabular}

EBIA-G: EBIA Guarani; TI: Terra Indígena.

* Aldeias que não participaram da fase qualitativa do estudo. 
dade e aprofundamento de estudos qualitativos e quantitativos entre indígenas, sem, contudo, aplicar a EBIA aos domicílios.

Por outro lado, o estudo de Vargas et al. 34 é o único que não usa o grupo focal, passando do painel de especialistas à aplicação da "EBIA modificada" aos domicílios, auxiliada pelos AIS. Reparando o constrangimento dos indígenas em falar sobre o tema, o estudo considera que o uso da EBIA deve ser adaptado às especificidades de cada povo, sem apresentar as possíveis discussões conceituais e seus impactos sobre o instrumento aplicado aos Mbya do Espírito Santo.

Dentre os estudos selecionados, aqueles sobre a adaptação da EBIA entre os Guarani de São Paulo são os mais longevos. Compreendem diferentes tipos de publicações, variando mais em grau de aprofundamento da adaptação do instrumento em capturar diferentes aspectos da insegurança alimentar, do que do ponto de vista das aldeias estudadas e metodologias empregadas 10,25,26,33. A publicação mais recente versa sobre uma das três fases dos estudos de validação da EBIA para os Guarani, a EBIA-G, desenvolvida ao longo de cinco anos 25 .

Os estudos sobre a fase qualitativa da validação duraram dois anos e não haviam sido publicados até 2018, registram os autores. Sobretudo por intermédio de três grupos focais, conversas informais, visitas e contato com lideranças e profissionais não especificados quanto ao pertencimento étnico, essa fase identificou como os Guarani definiam o construto da segurança alimentar e seus elementos-chave 25 . Os conceitos, conhecimentos e percepções Guarani foram equacionados e organizados segundo as dimensões da insegurança alimentar consideradas universais e dispostos gradualmente: da incerteza sobre a disponibilidade de comida em um momento próximo e o comprometimento da qualidade da alimentação às estratégias domiciliares para gerir a insegurança alimentar 25 .

Outros trabalhos relacionados ao desenvolvimento da EBIA-G descrevem metodologia, aldeias, fases e objetivos das pesquisas. A possibilidade de se chegar a uma EBIA-G, como registram, significaria também a possibilidade de construir uma EBIA-I (indígena), aplicada caso a caso, na direção de atingir uma escala única e nacionalmente utilizável como instrumento de governança nas políticas públicas direta ou indiretamente relacionadas ao aprimoramento das condições de alimentação e nutrição de povos indígenas 10,26.

Os estudos publicados em 2009 10,33, 201026 e 201825 compreendem as mesmas aldeias, sendo constituídos por um painel de especialistas em condições de alimentação e nutrição de diferentes povos indígenas, entre os quais os Guarani. No estudo de 2018, dessas discussões derivou uma primeira adaptação da EBIA, com 11 itens em português, posteriormente traduzidos ao Guarani.

As três publicações descrevem um total de três grupos focais, com as mesmas características, compostos por homens adultos, mulheres adultas e jovens. O estudo de 201825 descreve que os métodos dos grupos focais foram "adaptados à cultura Guarani", sem, no entanto, detalhar as aldeias participantes, o número de integrantes ou onde aconteceram. No caso do relatório de pesquisa, publicado em 2009 33, os três grupos focais aconteceram em uma pousada, em ambiente considerado pelos pesquisadores semelhante ao da aldeia e propício à concentração dos participantes nas discussões. Participaram 20 indivíduos pertencentes a uma das quatro aldeias participantes da pesquisa 33 . A publicação de 2010 não menciona grupos focais, mas entrevistas com membros de uma das aldeias 26 .

Boa parte dos ajustes realizados ao longo do processo de validação da EBIA-G aconteceu por intermédio de contatos do grupo de pesquisa e especialistas não indígenas com lideranças e com o entrevistador indígena que, tendo sido selecionado segundo critérios de bilinguismo e capacidade de compreensão do estudo, acompanhou as diversas fases e reuniões da pesquisa sediadas na Universidade Estadual de Campinas 25. Aplicada por esses entrevistadores, a EBIA-G passou por diferentes adaptações e testagens até atingir a sua versão final. Dos 15 itens originais da EBIA, chegou-se a uma versão validada final, com 6 itens, traduzidos ao Guarani 25.

Por um lado, os autores assinalam que as comunidades participantes não são "representativas" de outras comunidades Guarani ou de povos indígenas do país. Por outro, atribuem ao êxito da validação da EBIA-G a possibilidade de desenvolvimento de uma EBIA Indígena (EBIA-I), por meio de testes entre outros povos indígenas do Brasil 25. É reafirmada a intenção de que a EBIA-I sirva à governança de gestores, aprimorando o sucesso em identificar grupos e avaliar impactos das iniciativas de amenização da insegurança alimentar de um dos segmentos mais vulneráveis do país 25 . 


\section{A EBIA e a sociodiversidade indígena no Brasil: algumas reflexões}

Entre as décadas de 1980 e 1990, as ideias sobre a diversidade étnica e cultural da composição populacional de diferentes países da América Latina ganharam expressão no corpus jurídico de suas Constituições Nacionais 36,37. Como em outros países sul-americanos, na década de 90 foram criados programas e ações de saúde direcionados aos povos indígenas que, ao menos juridicamente, incorporam noções de respeito às diferenças socioculturais, inclusive na produção de informações 38 , mais tarde estendidas a outras políticas públicas relativas a indígenas 39 .

Com relação à adequação da EBIA aos diferentes povos indígenas do país, isto implica compatibilizar o instrumento às especificidades de suas plurais visões de mundo, formas de organização social e noções de "condições de vida" ideais. As iniciativas devem dialogar com concepções nativas sobre sua alimentação, o que inclui avaliar se há e qual o sentido de noções caras às escalas, como "qualidade" e "quantidade" de comida. Esses conceitos têm sido detalhadamente analisados em trabalhos do campo da etnologia e antropologia da alimentação e nutrição de povos indígenas, alguns destes contando, também, com análises sobre consumo alimentar, sob uma perspectiva, digamos, biomédica. Ainda que específicos, os estudos apontam para questões a serem consideradas, reafirmando a miríade de formas pelas quais esses povos lidam com a alimentação e tudo o que a cerca 40,41,42.

Entre outras questões relevantes às formas de acesso a alimentos pelos domicílios indígenas, o dinamismo de seus sistemas alimentares e a sazonalidade devem ser especialmente considerados. Submetidos a crescentes pressões sobre seus territórios e, em muitos casos, à monetarização de suas economias alimentares, os sistemas alimentares nativos seguem em franca transformação 40 . A sazonalidade tem sido raramente investigada 40,43 , dimensão ausente também do desenho e da discussão das principais pesquisas relacionadas à EBIA, conforme demonstramos. O trabalho de Yuyama et al. 31 registra a importância da variável, que não foi efetivamente incorporada em iniciativas posteriores.

Nas discussões acerca de sua aplicação, parte da experiência de "(in)segurança alimentar" e da "fome" é entendida como universal e mensurável, ainda que em contextos de reconhecida singularidade. Contemplar a sociodiversidade indígena e ao mesmo tempo garantir a comparabilidade entre os diversos contextos etnográficos é também, parafraseando Azevedo et al. 10, um desafio que precisa ser enfrentado na busca por uma compreensão culturalmente sensível do fenômeno e, parece-nos, um dos mais significativos.

Uma vez aceita a possibilidade de haver elementos comuns, mensuráveis e comparáveis nas experiências de insegurança alimentar de povos plurais, o que fica de fora e poderia ser importante a esta compreensão, especialmente com base na perspectiva nativa? Autores como Coates et al. 20 não deixam de aderir à ideia de que há muitos fatores comuns à experiência de insegurança alimentar, entre diversos povos e culturas no mundo. Mas também se perguntam, justamente, sobre o que essas coisas "comuns" podem deixar de "medir" ou "expressar", uma possibilidade mesmo onde o uso das escalas demonstra ser internamente consistente.

Centrar análises sobre aspectos comuns e comparáveis, uma condição operativa do modelo escalar, pode conduzir a evitar ambiguidades e, ao mesmo tempo, a que redundâncias, talvez significativas da perspectiva nativa, sejam extraídas. Para capturar o universal na experiência da fome, justamente as particularidades daquela experiência vão sendo apagadas. A diversidade dos sistemas alimentares pode acabar sendo subtraída, "purificada" 44 , no que talvez eles tenham de mais relevante do ponto de vista dos povos sobre os quais pretendemos conhecer e mensurar a experiência com sua comida e com a "fome".

$\mathrm{Na}$ relação entre epistemologias possivelmente distintas, corre-se o risco do diálogo entre os conhecimentos nativo e científico assumir uma assimetria persistente, com predominância de um em detrimento de outro 44, apesar da franca disposição dos pesquisadores em dialogar. Afinal, as escalas de insegurança alimentar foram originalmente criadas com base na "nossa" compreensão de eventos e situações selecionados e interpretados como relevantes ao constructo da insegurança alimentar e da "fome".

Como afirmam Webb et al. 21, o "acesso à comida" demonstrou ser um conceito relativo à experiência de insegurança alimentar dos domicílios americanos. Porque proveniente desse universo, sua compreensão prescinde mesmo da privação alimentar efetiva para existir entre os entrevistados. Nesse caso, o comprometimento do acesso à comida explicava melhor o estado nutricional das pessoas 
do que fatores como o acesso à atenção primária em saúde, à potabilidade da água ou ao saneamento adequado ${ }^{21}$. Isso implica assumir também que pode haver distintas relações entre renda e insegurança alimentar onde a produção de alimentos pelo próprio domicílio é significativa 21.

Por sua vez, a importância da "disponibilidade" e do "acesso físico e econômico" aos alimentos está referida a uma epistemologia que associa o comprometimento gradativo deste acesso à piora das "condições de vida" domiciliares. A possibilidade de aquisição, armazenamento e gestão do acúmulo de comida, ao longo de um determinado período, são critérios centrais neste modelo. Mas o que significaria ou como se dá o "acesso" a alimentos no universo múltiplo de povos indígenas brasileiros?

Ainda que consistências possam estar fortemente expressas no valor de face das escalas de insegurança alimentar, sua adequação a cada um dos contextos socioculturalmente diversos de aplicação não pode prescindir de uma igualmente forte articulação com dados etnográficos. Do contrário, mesmo medidas consideradas mais robustas e consistentes poderão ter somente uma frágil conexão com a realidade analisada.

\section{Escalas, povos indígenas e governança}

Diante da reconhecida gravidade da situação alimentar e nutricional indígena no país, a adoção de uma escala única para avaliação e governança deve obedecer a um processo amplo, duradouro, interdisciplinar e, notadamente, participativo de discussão nacional, dialogando tanto com a sociodiversidade quanto com noções de bem-estar e saúde possivelmente diversas. O debate envolveria não apenas uma reflexão sobre o sentido que o conceito de insegurança alimentar possa ter para suas epistemologias de mundo, mas também a quais tipos de ações esta aplicação poderá conduzir. O conceito de segurança alimentar, afinal, tem sido alvo de preocupação de organizações indígenas, integrando levantamentos participativos e constituindo, mesmo, um eixo temático das duas últimas Conferências Nacionais de Saúde Indígena 45,46,47.

Além de reconhecer a importância do problema, trata-se de incluir outras variáveis em sua compreensão, de modo a produzir soluções que tenham efetividade e, ao mesmo tempo, respeitem a diversidade dos modos de vida de povos indígenas, constitucionalmente prevista no Brasil. Reconhecendo as múltiplas dimensões da insegurança alimentar, Webb et al. 21 mencionam que a usual associação da "fome" com a falta de disponibilidade de comida, e da falta de disponibilidade de comida com a produção de mais comida, conduziu a uma hiperconfiança em soluções de agricultura doméstica, para problemas que possivelmente tinham outras origens. Iniciativas inspiradas em diagnósticos fundados em um modo semelhante de conceber a vida e os problemas de povos indígenas no país têm se multiplicado ao longo da história. Projetadas para garantir e aumentar a produção de alimentos, seu excedente, gerar renda a partir dele ou gerar alimento por meio de políticas de transferência de renda, elas não têm alcançado resultados satisfatórios entre povos indígenas brasileiros 48 .

Frequentemente, indígenas, suas formas de vida ou sua "incapacidade” em gerar "produção", "excedente" ou "renda" são responsabilizados por condições desiguais de saúde, saneamento ambiental, degradação e limitação de seus territórios, histórica e juridicamente da competência do Estado. Intencionalmente ou não, a causa dos problemas tem seu eixo completamente deslocado. Mais recentemente, a política indigenista oficial reacende projetos constitucionalmente desautorizados de "integração" de povos indígenas à economia de mercado, como forma de usurpar ou ocupar indevidamente seus territórios, abrindo-os a atividades de mineração e ao agronegócio 49, ambos estimulados por ações de uma sucessão de Governos e, sem precedentes, pelo Governo atual.

Um estudo interdisciplinar sobre a percepção, a execução e o uso de recursos provenientes de programas nacionais relacionados à segurança alimentar e nutricional entre povos indígenas, entre os Shawi peruanos, demonstra quanto o respeito aos contextos culturais nativos - de suas configurações territoriais, histórico de contato a dietas e sistemas alimentares - é decisivo à capacidade das iniciativas em efetivamente promover suas condições de saúde 50. Em suas conclusões, os autores acrescentam outra dimensão importante ao aspecto da eficiência sanitária dos Programas. Considerar as especificidades de indígenas é, além do mais, um "imperativo moral" que atende resolutivamente às iniquidades de suas condições de saúde naquele país 50 . 
No Brasil, onde também persistem as iniquidades históricas, em especial, de suas condições de saúde, esse imperativo moral está reforçado pelo imperativo jurídico de que as políticas públicas destinadas ao segmento adotem tanto formas de diagnóstico quanto soluções culturalmente sensíveis. Colocando dessa forma, o respeito à sociodiversidade de povos indígenas atende a um imperativo simultaneamente moral e jurídico, mas que não deixa de estar também relacionado à eficiência das políticas públicas a eles destinadas.

\section{Conclusões}

A produção de informações e o esforço de "tornar visíveis" os povos indígenas, suas condições sociodemográficas e de saúde, têm recomendado sua participação e de suas organizações, de modo que seus "valores, conceitos de saúde e prioridades" possam resultar na expressão e mensuração adequada de suas "noções de bem-estar e saúde" 2. Esse é um passo importante à correção das assimetrias do diálogo entre povos indígenas, seus sistemas alimentares e especialistas. No caso específico de uma possível EBIA-I, estudos pautados em etnografia e com profundidade temporal poderiam sugerir caminhos e percepções outras sobre esses povos e sua comida, fundados em outras epistemologias e conceitos que não aqueles adotados pelos Estados-nação. A participação de povos indígenas nesse processo envolveria, inclusive, a possibilidade de refletir sobre se e, em caso positivo, qual sentido a própria noção de "segurança alimentar" tem diante de suas epistemologias alimentares.

Mais precisamente, propomos que estudos etnográficos constituam componentes específicos da metodologia transdisciplinar dedicada à compreensão dos fenômenos da insegurança alimentar e da fome em contextos indígenas, na direção de um diálogo intercultural o mais simétrico possível. Os elementos aqui apontados, entre outros que possam emergir de um conhecimento progressivo das dinâmicas e perspectivas desses povos sobre a insegurança alimentar, devem ser considerados nas futuras iniciativas para compreender e medir a experiência da insegurança alimentar entre povos indígenas no país.

Por fim, faz-se necessário pensar criticamente quais tipos de ações estatais podem resultar da aplicação das Escalas de Insegurança Alimentar entre esses povos. Essa observação é válida tanto para o desenvolvimento de instrumentos de governança que sejam potencialmente capazes de atingir diretamente sua vida, gerando novas ações ou correções, como para a (re)formulação de políticas públicas às quais já tenham acesso.

\section{Colaboradores}

Ambos os autores participaram da concepção do projeto, análise e interpretação dos dados, redação do manuscrito, revisão crítica relevante do conteúdo intelectual e aprovação final da versão a ser publicada.

\section{Informações adicionais}

ORCID: Adriana Romano Athila (0000-0001-7441380X); Maurício Soares Leite (0000-0002-4183$375 \mathrm{X})$

\section{Agradecimentos}

A João Luiz Dornelles Bastos e Deise Bresan, pelos ricos diálogos sobre o tema. Ao Programa de Pós-graduação em Epidemiologia em Saúde Pública da Escola Nacional de Saúde Pública Sergio Arouca, Fundação Oswaldo Cruz, pela bolsa de pós-doutorado (Coordenação de Aperfeiçoamento de Pessoal de Nível Superior - bolsista de PNPD/Capes). À Fundação Wellcome Trust (203486/Z/16/Z). 


\section{Referências}

1. Gracey M, King M. Indigenous health part 1: determinants and disease patterns. Lancet 2009; 374:65-75.

2. Anderson I, Robson B, Connolly M, Al-Yaman F, Bjertness E, King A, et al. Indigenous and tribal peoples' health (The Lancet - Lowitja Institute Global Collaboration): a population study. Lancet 2016; 388:131-57.

3. Coimbra Jr. CEA, Santos RV, Cardoso AM, Souza MC, Garnelo L, Rassi E, et al. The First National Survey of Indigenous People's Health and Nutrition in Brazil: rationale, methodology, and overview of results. BMC Public Health 2013; 13:1-19.

4. Bresan D, Bastos JL, Leite MS. Epidemiologia da hipertensão arterial em indígenas Kaingang, Terra Indígena Xapecó, Santa Catarina, Brasil, 2013. Cad Saúde Pública 2015; 31:331-44.

5. Leite MS. Nutrição e alimentação em saúde indígena: notas sobre a importância e a situação atual. In: Garnelo L, Pontes AL, organizadores. Saúde indígena: uma introdução ao tema. Brasília: Secretaria de Educação Continuada, Alfabetização, Diversidade e Inclusão, Ministério da Educação; 2012. p. 156-83.

6. Horta BL, Santos RV, Welch JR, Cardoso AM, Santos JV, Assis AM, et al. Nutritional status of indigenous children: findings from the First National Survey of Indigenous People's Health and Nutrition in Brazil. Int J Equity Health 2013; 12:23.

7. Fávaro TR, Ferreira AA, Cunha GM, Coimbra Jr. CEA. Excesso de peso em crianças indígenas Xukuru do Ororubá, Pernambuco, Brasil: magnitude e fatores associados. Cad Saúde Pública 2019; 35 Suppl 3:e00056619.

8. Leite MS, Cardoso AM, Coimbra Jr. CEA, Welch JR, Gugelmin SA, Lira PIC, et al. Prevalence of anemia and associated factors among indigenous children in Brazil: results from the First National Survey of Indigenous People's Health and Nutrition. Nutr J 2013; 12:69.

9. Borges MC, Buffarini R, Santos RV, Cardoso AM, Welch JR, Garnelo L, et al. Anemia among indigenous women in Brazil: findings from the First National Survey of Indigenous People's Health and Nutrition. BMC Womens Health 2016; 16:1-12.

10. Azevedo MM, Segall-Corrêa AM, Ferreira MBR. Estudo do Conceito e Percepção de Segurança Alimentar e Nutricional entre os Guarani no Estado de São Paulo. In: Mendes RT, Vilarta R, Gutierrez GL, organizadores. Qualidade de vida e cultura alimentar. Campinas: Ipês Editorial; 2009. p. 167-76.

11. Kepple AW, Segall-Corrêa AM. Conceituando e medindo segurança alimentar e nutricional. Ciênc Saúde Colet 2011; 16:187-99.

12. Radimer KL, Olson CM, Campbell CC. Development of indicators to assess hunger. J Nutr 1990; 120 Suppl 11:1544-8.

13. Radimer KL. Measurement of household food security in the USA and other industrialized countries. Public Health Nutr 2002; 5:859-64.
14. Frongillo Jr. EA. Validation of measures of food insecurity and hunger. J Nutr 1999; 129 Suppl 2:506s-9.

15. Brasil. Lei no 11.346 , de 15 de setembro de 2006. Cria o Sistema Nacional de Segurança Alimentar e Nutricional - SISAN com vistas em assegurar o direito humano à alimentação adequada e dá outras providências. Diário Oficial da União 2006; 18 set.

16. Segall-Corrêa AM, Marin-Leon L. A segurança alimentar no Brasil: proposição e usos da escala brasileira de medida da insegurança alimentar (EBIA) de 2003 a 2009. Segurança Alimentar e Nutricional 2009; 16:1-19.

17. Ministério do Desenvolvimento Social e Combate à Fome. Escala Brasileira de Insegurança Alimentar - EBIA: análise psicométrica de uma dimensão da Segurança Alimentar e Nutricional. Brasília: Ministério do Desenvolvimento Social e Combate à Fome; 2014. (Estudo Técnico 1).

18. Instituto Brasileiro de Geografia e Estatística. Pesquisa Nacional por Amostra de Domicílios. Segurança Alimentar 2004/2009. Rio de Janeiro: Instituto Brasileiro de Geografia e Estatística; 2010.

19. Ministério da Saúde. Pesquisa Nacional de Demografia e Saúde da Criança e da Mulher PNDS 2006: dimensões do processo reprodutivo e da saúde da criança. Brasília: Ministério da Saúde; 2009. (Série G. Estatística e Informação em Saúde).

20. Coates J, Frongillo EA, Rogers BL, Webb P, Wilde PE, Houser R. Commonalities in the experience of household food insecurity across cultures: what are measures missing? J Nutr 2006; 136:1438S-48S.

21. Webb P, Coates J, Frongillo Jr. EA, Rogers BL, Swindale A, Bilinsky P. Measuring household food insecurity: why it's so important and yet so difficult to do. J Nutr 2006; 136:1404S-8S

22. Melgar-Quinonez H, Hackett M. Measuring household food security: the global experience. Rev Nutr 2008; 21 Supp 1:27s-37s.

23. Fávaro T, Ribas DLB, Zorzatto JR, Segall-Corrêa AM, Panigassi G. Segurança alimentar em famílias indígenas Teréna, Mato Grosso do Sul, Brasil. Cad Saúde Pública 2007; 23:785-93.

24. Franceschini T. O direito humano à alimentação adequada e à nutrição do povo Guarani e Kaiowá: um enfoque holístico - resumo executivo. Brasília: FIAN Brasil; 2016.

25. Segall-Correa AM, Marin-Leon L, Azevedo MMA, Ferreira MBRR, Gruppi DR, Camargo DFM, et al. The Brazilian food security scale for indigenous Guarani households: development and validation. Food Security 2018; 10:1547-59.

26. Segall-Correa AM, Azevedo MM, Ferreira B, Kepple AW, León-Marin L. Perception of food insecurity among indigenous Guarani communities in the state of São Paulo, Brasil. The FASEB Journal 2010; 24 Suppl 1. https://www. fasebj.org/doi/abs/10.1096/fasebj.24.1_sup plement.104.6. 
27. Merry SE. The seductions of quantification: measuring human rights, gender violence, and sex trafficking. Chicago/London: The University of Chicago Press; 2016.

28. Santos RV, Guimarães BN, Simoni AT, Silva LO, Oliveira Antunes M, Souza Damasco F, et al. The identification of the Indigenous population in Brazil's official statistics, with an emphasis on demographic censuses. Stat J IAOS 2019; 35:29-46.

29. Santos RV, Bastos JL, Cruz OG, Longo LAFB, Flowers NM, Pereira NOM. Parity of Indigenous and non-Indigenous women in Brazil: Does the reported number of children born depend upon who answers national census questions? Plos One 2015; 10:e0123826.

30. Brasil. Decreto no 7.272, de 25 de agosto de 2010. Regulamenta a Lei no 11.346 , de 15 de setembro de 2006, que cria o Sistema Nacional de Segurança Alimentar e Nutricional - SISAN, institui a Política Nacional de Segurança Alimentar e Nutricional - PNSAN, estabelece os parâmetros para a elaboração do Plano Nacional de Segurança Alimentar e Nutricional, e dá outras providências. Diário Oficial da União 2010; 26 ago.

31. Yuyama LKO, Py-Daniel V, Ishikawa NK, Medeiros JF, Kepple AW, Segall-Corrêa AM. Percepção e compreensão dos conceitos contidos na Escala Brasileira de Insegurança Alimentar, em comunidades indígenas no Estado do Amazonas, Brasil. Rev Nutr 2008; 21 Suppl:53s-63s.

32. Instituto Brasileiro de Geografia e Estatística. Censo Demográfico 2010: Características Gerais dos Indígenas. Resultados do Universo. Rio de Janeiro: Instituto Brasileiro de Geografia e Estatística; 2012.

33. Segall-Corrêa AM, Azevedo M, Marín L, Ferreira MB, Kepple AW, Panigassi G, et al. Estudo dos conceitos, conhecimentos e percepções sobre segurança, insegurança alimentar e fome em quatro grupos de etnia Guarani no Estado de SP. Campinas: Universidade Estadual de Campinas; 2009.

34. Vargas LC, Souza RS, Sufiate CB, Santos EM, Sipioni ME, Rezende AMB. Segurança Alimentar e Nutricional entre os Guaranis Mbyá da Aldeia Boa Esperança, Aracruz, Espírito Santo, Brasil. RASBRAN: Revista da Associação Brasileira de Nutrição 2013; 5:5-12.

35. Silveira NH, Melo CR, Jesus SC, Organizadores. Diálogos com os Guarani: articulando compreensões antropológicas e indígenas. Florianópolis: Editora da Universidade Federal de Santa Catarina; 2016.

36. Sieder R, Organizador. Multiculturalism in Latin America: indigenous rights, diversity, and democracy. New York: Palgrave Macmillan; 2002.

37. Hooker J. Indigenous inclusion/black exclusion: race, ethnicity and multicultural citizenship in Latin America. J Lat Am Stud 2005; 37:285-310.
38. Ferreira LO. Interculturalidade e saúde indígena no contexto das políticas públicas brasileiras. In: Langdon EJ, Cardoso MD, organizadores. Saúde indígena: políticas comparadas na América Latina. Florianópolis: Editora da UFSC; 2015. p. 217-46.

39. Shankland A, Athias R. Decentralisation and difference: indigenous peoples and health system in the brazilian amazon. IDS Bulletin 2006; 38:77-88.

40. Leite MS. Transformação e persistência: antropologia da alimentação e nutrição em uma sociedade indígena Amazônica. Rio de Janeiro: Editora Fiocruz; 2007.

41. van Velthem LH. Comer verdadeiramente: produção e preparação de alimentos entre os Wayana. Horizontes Antropológicos 1996; 4:10-26.

42. Silveira NH. Culinária mbya, um modo da persistência guarani. In: Silveira NH, Melo CR, Jesus SC, organizadores. Diálogos com os Guarani: articulando compreensões antropológicas e indígenas. Florianópolis: Editora da UFSC; 2016. p. 119-39.

43. Flowers NM. Seasonal factors in subsistence, nutrition, and child growth in a Central Brazilian community. In: Hames RB, Vickers WT, editors. Adaptive responses of native Amazonians. New York: Academic Press; 1983. p. 357-90.

44. Latour B. Ciência em ação. São Paulo: Editora Unesp; 2000.

45. Federação das Organizações Indígenas do Rio Negro. FUNAI, ISA E FOIRN assinam acordo de cooperação técnica para fortalecer a gestão indígena sobre territórios do Rio Negro. https://foirn.blog/2016/05/12/funai-isa-e-foir$\mathrm{n}$-assinam-acordo-de-cooperacao-tecnica-ac$\mathrm{t}$-para-fortalecer-a-gestao-indigena-sobre-territorios-do-rio-negro/ (acessado em 01/ Mai/2020).

46. Fundação Nacional de Saúde. 4a Conferência Nacional de Saúde Indígena. Relatório final. Brasília: Fundação Nacional de Saúde; 2007.

47. Ministério da Saúde. 5a Conferência Nacional de Saúde Indígena. Relatório final. Brasília: Ministério da Saúde; 2015.

48. Athila AR. "Gente que anda" e os percursos da "cidadania". In: Ricardo F, Ricardo B, organizadores. Povos indígenas no Brasil: 2011/2016. São Paulo: Instituto Socioambiental; 2017. p. 236-9.

49. Carneiro da Cunha M, Caixeta R, Campbell JM, Fausto C, Kelly JA, Lomnitz C, et al. Indigenous peoples boxed in by Brazil's political crisis. Journal of Ethnographic Theory 2017; 7:403-26.

50. Zavaleta C, Berrang-Ford L, Llanos-Cuentas A, Cárcamo C, Forda J, Silvera R, et al. Indigenous Shawi communities and national food security support: right direction, but not enough. Food Policy 2017; 73:75-87. 


\section{Abstract}

The Brazilian Food Insecurity Scale (EBIA) was validated for the Brazilian context in 2004. Since then, the scale has evaluated and measured the experience of food insecurity in rural and urban households, initially in the Brazilian National Household Sample Survey and later in the Brazilian National Survey of Demographic and Health. However, indigenous peoples have not been examined specifically in these surveys, despite recognition of their food and nutritional vulnerability. In this scenario, we analyze and discuss the application of the EBIA among indigenous peoples in Brazil, based on a set of fundamental questions for understanding and measuring their experience with food insecurity and "hunger". We conduct a sociopolitical and ethnographic analysis of a set of official documents and significant articles on the use of psychometric scales of food insecurity among Brazilian indigenous peoples, compared to international studies on the validation and application of scales in other sociocultural contexts. The initiatives with adaptation and application of the EBIA to indigenous peoples in Brazil indicate that understanding and measuring food insecurity in these peoples poses a major challenge. Particularly complex is the proposal to guarantee comparability of different contexts while taking into account the multiple local singularities. We propose that ethnographic studies should serve as specific components of future initiatives on this topic and that they should cover aspects such as the seasonality of indigenous peoples' food production, different processes of food monetization, and the dynamics of their food systems.

Medical Anthropology; Public Policy; Populations Groups; Food Insecurities

\section{Resumen}

Validada para el contexto nacional brasileño en 2004, la Escala Brasileña de Inseguridad Alimentaria (EBIA) ha evaluado y calculado desde entonces la existencia de inseguridad alimentaria en domicilios rurales y urbanos, inicialmente mediante la Encuesta Nacional por Muestra Domiciliaria $y$, posteriormente, a través de la Encuesta Nacional de Demografía y Salud. No obstante, los pueblos indígenas no fueron específicamente examinados en estas encuestas, a pesar del reconocimiento de su vulnerabilidad alimentaria y nutricional. En este escenario, analizamos $y$ discutimos la aplicación de la EBIA entre pueblos indígenas del país, a partir de un conjunto de cuestiones consideradas aquí fundamentales para la comprensión y medición de su experiencia de inseguridad alimentaria y "hambre". Se realiza un análisis sociopolítico y etnográfico de un conjunto de documentos oficiales, así como de artículos significativos sobre el uso de escalas psicométricas de inseguridad alimentaria entre pueblos indígenas brasileños, en contraste con artículos internacionales sobre la validación y aplicación de las escalas en otros contextos socioculturales. Las iniciativas de adaptación y aplicación de la EBIA en contextos indígenas brasileños indican que comprender y medir la inseguridad alimentaria entre estos pueblos es un desafío de magnitud considerable. Particularmente compleja es la propuesta de garantizar la comparabilidad entre contextos distintos, sin dejar de considerar las plurales singularidades locales. Proponemos que los estudios etnográficos establezcan componentes específicos para las futuras iniciativas dedicadas a este tema, así como que contemplen aspectos como: estacionalidad de la producción de alimentos, sus procesos diferenciales de monetarización, y dinamismo de sus sistemas alimentarios.

Antropología Médica; Política Pública; Grupos de Población; Seguridad Alimentaria
Recebido em 24/Out/2019

Versão final reapresentada em 07/Mai/2020 Aprovado em 14/Mai/2020 